\title{
Direct Laser Writing of Three-Dimensional Photonic Crystals with a Complete Photonic Bandgap in Chalcogenide Glasses**
}

\author{
By Sean Wong, Markus Deubel, Fabian Pérez-Willard, Sajeev John, Geoffrey A. Ozin,* \\ Martin Wegener, and Georg von Freymann*
}

The concept of three-dimensional (3D) photonic crystals (PCs) and photonic bandgap materials ${ }^{[1,2]}$ was introduced nearly twenty years ago. However, the fabrication of highquality 3D structures for the optical regime still remains a challenge. Three techniques have been discussed: i) direct semiconductor fabrication by using a layer-by-layer (LbL) method, combining high-precision alignment and wafer-fusion techniques, ${ }^{[3-6]}$ ii) templating by means of self-assembly of low-refractive-index colloidal microspheres, ${ }^{[7,8]}$ and iii) holographic laser lithography ${ }^{[9,10]}$ and direct laser writing $(\mathrm{DLW})^{[11,12]}$ to fabricate large-area, defect-free polymer templates. The latter two approaches require subsequent inversion $^{[13-15]}$ or double inversion ${ }^{[16,17]}$ steps with high-refractiveindex materials. Here we present a novel approach, namely DLW in all-inorganic, high-refractive-index chalcogenide glasses. This approach combines the flexibility of DLW with the benefits of a direct fabrication method, hence eliminating the need for subsequent inversion. The fabrication of wood-

[*] Prof. G. A. Ozin, Dr. G. von Freymann, S. Wong Materials Chemistry Research Group, Department of Chemistry University of Toronto

80 St. George Street, Toronto, ON M5S $3 \mathrm{H} 6$ (Canada)

E-mail: gozin@chem.utoronto.ca

Dr. G. von Freymann, S. Wong, M. Deubel, Prof. M. Wegener Institut für Nanotechnologie

Forschungszentrum Karlsruhe in der Helmholtz-Gemeinschaft D-76021 Karlsruhe (Germany)

E-mail: freymann@int.fzk.de

Dr. G. von Freymann, S. Wong, M. Deubel, Prof. M. Wegener Institut für Angewandte Physik, Universität Karlsruhe (TH) D-76128 Karlsruhe (Germany)

Dr. G. von Freymann, S. Wong, M. Deubel, Dr. F. Pérez-Willard, Prof. M. Wegener

DFG-Center for Functional Nanostructures (CFN)

Universität Karlsruhe $(\mathrm{TH})$

D-76128 Karlsruhe (Germany)

Prof. S. John, Dr. G. von Freymann

Department of Physics, University of Toronto

60. St. George Street, Toronto, ON M5S 1 A7 (Canada)

[**; GAO and SJ are Government of Canada Research Chairs. We are grateful to the Natural Sciences and Engineering Research Council of Canada (NSERC) for financial support of this work. We acknowledge the support by the Center for Functional Nanostructures (CFN) of the Deutsche Forschungsgemeinschaft (DFG) within project A.1.4. The research of G.v.F. is further supported by DFGprojects FR 1671/2-1 and FR 1671/4-3 (Emmy-Noether program) and that of M.W. by We 1497/9-1. pile structures ${ }^{[18]}$ with a complete gap of $3.5 \%$ takes less than two hours.

There are two stringent requirements for materials to be suitable for the fabrication of 3D PCs with an omni-directional photonic bandgap (PBG): i) the material has to be transparent over the wavelength region in which the $\mathrm{PC}$ is operated, and ii) the index of refraction should allow for an index contrast of at least 1.9 in the final structure to open the PBG. ${ }^{[18]}$ Unfortunately, most of the materials that fulfill requirement (i) do not comply with requirement (ii). This is especially awkward as most of the materials suitable for fabrication by using lithographic techniques like holographic laser lithography ${ }^{[9,10]}$ and direct laser writing ${ }^{[11,12]}$ belong to this group (i.e., all-organic photoresists). Prominent materials such as silicon or gallium arsenide, which fulfill both requirements, are however not suitable for 3D lithographic techniques. Therefore, these materials are usually incorporated by means of chemical vapor deposition (CVD) techniques into suitable templates, which are then removed at a later stage. ${ }^{[14,17]}$ Direct fabrication of 3D PCs in high-index materials seems preferable, but requires fabrication in a LbL assembly. ${ }^{[19]}$ Each layer has to be fabricated with a whole set of lithography and etching steps. ${ }^{[3-6]}$ Furthermore, ultra-precise alignment and wafer fusion for each additional layer is required at the cost of fabrication speed. Therefore, a technique combining both direct fabrication into a high-index material with the high flexibility, speed, and accuracy of direct laser writing is highly desirable.

Here, we present for the first time direct laser written 3D $\mathrm{PCs}$ with a PBG made from $\mathrm{As}_{2} \mathrm{~S}_{3}$ chalcogenide glasses. These materials are amorphous semiconductors with high transparency throughout the near-infrared and infrared spectral region. The index of refraction lies between 2.45 and 2.53 , ${ }^{[20]}$ sufficient to open a PBG. In $\mathrm{As}_{2} \mathrm{~S}_{3}$ the position of the absorption edge is found at $530 \mathrm{~nm}$ leaving a broad part of the visible spectrum accessible. Chalcogenide glasses are known for their photoinduced metastabilities, ${ }^{[21]}$ which have led to their widespread use in optical storage media like compact disk ROMs (CDRs) and digital versatile disks (DVDs). ${ }^{[21]}$ Chalcogenide glasses not only change their optical properties under photoexposure, but also their chemical properties. This allows for selective chemical etching, making them suitable candidates for lithographic techniques. Therefore, chalcogenide glasses have already been used for the fabrication of 3D PCs, but previous work has been limited to opal infiltration ${ }^{[22-24]}$ or LbL techniques, ${ }^{[5,6]}$ where two- or three-beam holographic 
laser lithography was combined with selective etching. As above-gap radiation and thus one-photon absorption was used in these experiments, the lithographic step could only be used to fabricate a single layer at a time. Consecutive layers had to be stacked on top of each other and PCs with a maximum of four layers have been reported. ${ }^{[5,6]}$

Here we use direct laser writing in $11 \mu \mathrm{m}$ thick $\mathrm{As}_{2} \mathrm{~S}_{3}$ films by means of two-photon absorption. Thin chalcogenide films are fabricated through thermal evaporation of glassy $\mathrm{As}_{2} \mathrm{~S}_{3}$ at temperatures below $390{ }^{\circ} \mathrm{C}$ and at low rates (see Experimental). This yields thin films, mainly composed of $\mathrm{As}_{4} \mathrm{~S}_{6}$ cage molecules $-\mathrm{a}$ so-called molecular glass (see Fig. 1, step I). ${ }^{[2,25,26]}$ A high content of $\mathrm{As}_{4} \mathrm{~S}_{6}$ is desirable, as $\mathrm{As}_{4} \mathrm{~S}_{6}$ was found to be the fragment, which gives the largest photopolymerization effect. ${ }^{[26]}$ Fast evaporation rates cause thermal

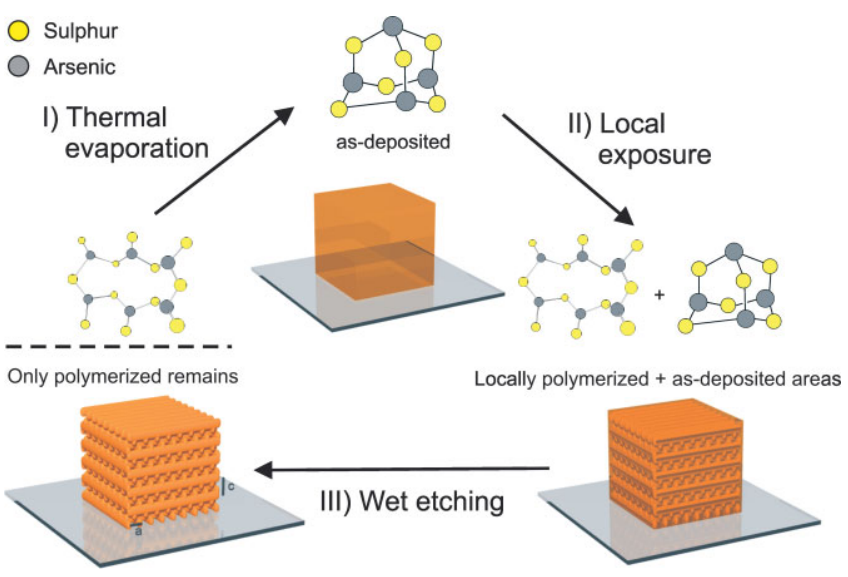

Figure 1. The three fabrication steps. 1) Thermal evaporation of glassy $\mathrm{As}_{2} \mathrm{~S}_{3}$ at $T<380^{\circ} \mathrm{C}$ with a rate of $6 \mathrm{~nm} \mathrm{~s}^{-1}$ yields a thin film of molecular glass composed of $\mathrm{As}_{4} \mathrm{~S}_{6}$ cage molecules. II) DLW locally exposes the material, initiating a topotactic ring-opening polymerization back into the glassy $\mathrm{As}_{2} \mathrm{~S}_{3}$. III) Removal of the unexposed molecular glass by means of wet etching reveals the 3D photonic crystal.

fragmentation of the precursor into a distribution of molecular fragments, mainly composed of $\mathrm{As}_{4} \mathrm{~S}_{4}$ cages and $\mathrm{S}_{8}$ rings. ${ }^{[27-29]}$ Such films are less effective for photopolymerization. ${ }^{[26]}$ Local photoexposure of the molecular glass initiates polymerization, transforming the $\mathrm{As}_{4} \mathrm{~S}_{6}$ cage molecules back into the original $\mathrm{As}_{2} \mathrm{~S}_{3}$ glass (Fig. 1, step II). ${ }^{[20]}$ As the stoichiometry and bond lengths between the cage cluster and the glass are the same, the movement of atoms during all process steps is kept minimal. This process is reminiscent of a topotactic ring-opening polymerization of $\mathrm{S}_{2} \mathrm{~N}_{2} \cdot{ }^{\left[{ }^{[0]}\right.}$ We observe neither shrinkage nor expansion of the written structure. This is an important advantage over photoresists, like the most commonly used SU-8, which shrinks approximately $7 \%$ during development, ${ }^{[10]}$ causing structural distortions.

For DLW we tune the laser to a wavelength with negligible one-photon absorption of the chalcogenide glass (see Experimental). Through tight focusing, the high intensity in the focal volume allows for photoinduced polymerization by means of a multiphoton absorption process. For focusing with an oilimmersion objective into an index-matched medium, the exposed volume pixels (so-called voxels) are of near ellipsoidal shape with a ratio between axial diameter/lateral diameter of about 2.7 (e.g., measured in SU-8 ${ }^{[12]}$ ) for the experimental conditions used. For focusing into a medium with significantly higher refractive index, a number of aspects have to be considered. i) The most striking aspect is the defocus. This means that an axial movement of the chalcogenide film, by means of the piezoelectric translation stage, of, e.g., $2 \mu \mathrm{m}$, leads to a much larger axial shift of the position of the exposed voxel. Under the experimental conditions used, the discrepancy between the actual and intended writing position is roughly a factor of two. Intuitively, in a ray-optics picture, the rays are refracted towards the surface normal inside the chalcogenide, moving the focus position deeper into the chalcogenide (see inset in Fig. 2a). This simplistic reasoning is confirmed by state-of-the-art numerical calculations of the intensity distribution, based on the vectorial Debye theory (Fig. 2a). ${ }^{[31]}$ The incident polarization is chosen along the $x$ direction. Here, we define the intensity, $I$, as the square modulus of the electricfield vector and plot the square of this intensity, which mimics the two-photon absorption process. The defocus becomes apparent from comparing the naively expected focus position (white dashed lines) with the actual focus position. ii) The refraction of rays towards the surface normal reduces the opening angle (see inset), and hence the effective numerical aperture of the focusing optics. This effect leads to a drastic elongation of the voxels from the above-mentioned factor of $2.7^{[12]}$ to about 8 for a focus close to the interface (see Fig. 2a). iii) Due to higher-order spherical aberrations, this elongation factor also depends on the axial focus position, as can be seen from Figure 2a. Furthermore, the shape of the voxels changes with increasing focus depth. In particular, side lobes emerge and eventually merge with the main maximum. As a direct consequence, the peak intensity and hence the two-photon absorption decreases. For example, the peak intensity $\left(I^{2}\right)$ decreases by as much as a factor of two for a focusing depth of $6 \mu \mathrm{m}$ (see Fig. 2b). The three aspects mentioned above are also found in corresponding experiments (Fig. 2c). Consequently, to implement DLW into chalcogenide glasses, counter measures have to be taken to overcome all three of these undesired aspects when working with high-index materials.

Generally, the following countermeasures could be taken. First, the apparent defocus can be corrected by choosing the proper writing depth according to the theoretically expected position of the maximum intensity. For moderate focusing depths, this corresponds to a constant factor. Second, several voxels have to be put side by side to correct for the wrong cross section of the resulting rods, reducing the aspect ratio of the rod cross section from eight to at least three. (According to our simulations, the latter elongation factor is the maximum value compatible with a complete PBG.) This step is exemplified in Figure 3a for a four-layer woodpile with a rod distance of $a=2 \mu \mathrm{m}$. The inset clearly shows four pairs of single-pass rods, resulting in an overall almost rectangular rod 
a

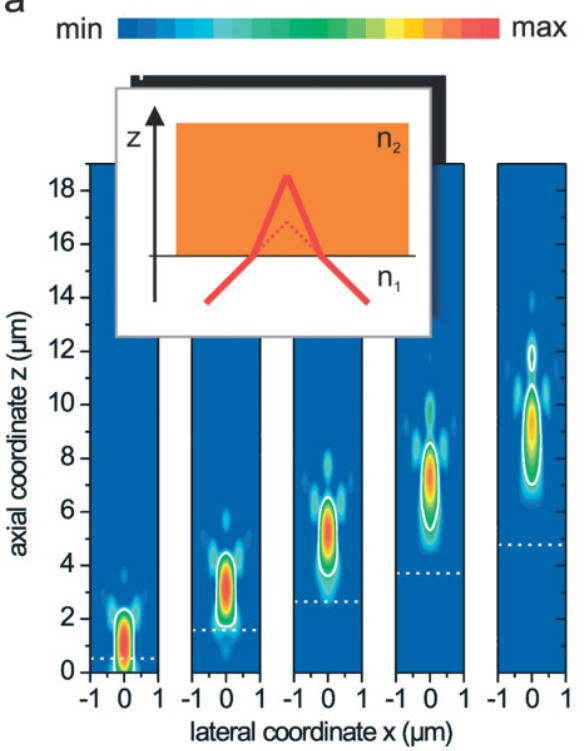

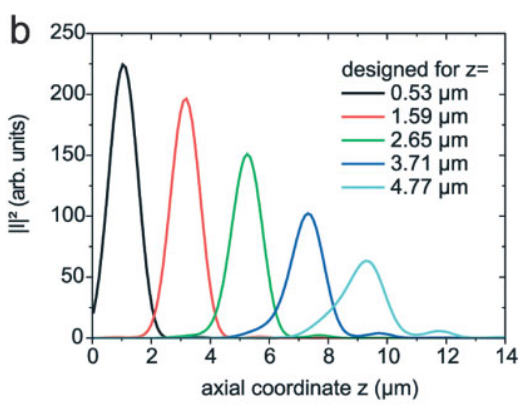

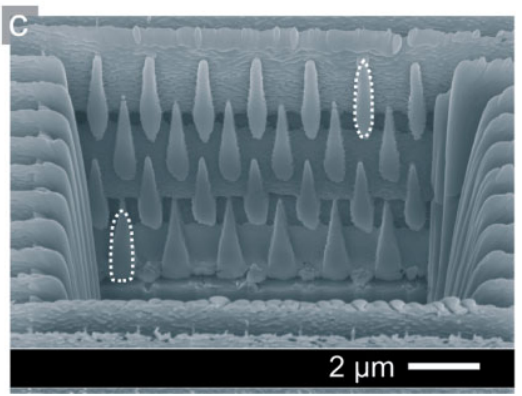

Figure 2. Influence of the index mismatch on the voxel shape. a) Two-photon intensity $|I|^{2}$ distribution near the focus calculated for different writing depths $d$. The interface is located at $z=0$. With increasing writing depth the intended writing position (indicated by the dashed white line) significantly deviates from the position of the intensity maximum as a result of defocusing (color coding on a logarithmic scale). The inset illustrates the origin of the defocus in a simplified geometrical picture for the transition from a medium with index $n_{1}$ to a medium with $n_{2}>n_{1}$. Additionally, voxels become more distorted owing to higher-order spherical aberrations (solid white lines are guides to the eyes). b) Cross sections of the intensity distributions in (a) along the $x=0 \mu \mathrm{m}$ axis. In addition to the distortions visible in (a) the maximum intensity in the focus significantly decreases with increased writing depth $d$. c) Focused-ion-beam cross section of an eight-layer woodpile written in a single pass per rod without any corrections. The asymmetric shape qualitatively resembles the calculated intensity distribution in (a) (dotted white lines are guides to the eye). Note that the horizontal and the vertical scales in (a) and (c) are directly comparable.

cross section. Third, an even more elaborate correction ${ }^{[32]}$ would be the introduction of additional optics into the system to precompensate the higher-order spherical aberrations introduced by the index mismatch and thus the decrease in intensity and the distortion of the voxel shape with increasing writing depth.

For a proof of concept of this strategy we limit ourselves to the first two correction steps and write woodpiles with a rod spacing of $a=1 \mu \mathrm{m}$. As these corrections cannot overcome the decrease in intensity according to (iii), only structures with a limited number of layers are possible. The defocus is corrected to yield the proper unit cell dimension of $c^{2} / a^{2}=2.0$ for face-centered-cubic translational symmetry. To correct the rod cross section, each rod was constructed of six single voxel lines, written $40 \mathrm{~nm}$ apart from each other, resulting in a rod width after etching of approximately $220 \mathrm{~nm}$ and an average aspect ratio of about 2.4 . The writing time for an eight-layer structure with a $22 \mu \mathrm{m} \times 22 \mu \mathrm{m}$ footprint is below $10 \mathrm{~min}$. The subsequent wet-etching step with a mixture of diisopentylamine in dimethylsulfoxide takes approximately $20 \mathrm{~min}$ (see Experimental). Figure $3 c$ shows a top view of the structure. Note the perfectly straight bars due to the negligible shrinkage. A focused-ion-beam cross section reveals the rod dimenrameters as discussed above, but different in three aspects, is illustrated in Figures 3 e,f. First, it is raised from the substrate so that the walls merely act as a mechanical support. Second, it is larger with four times the footprint. Third, it is thicker with twelve instead of eight layers. In sharp contrast to corresponding structures fabricated in SU- $8,{ }^{[12]}$ none of the images shown in Figure 3 exhibit any significant distortion of the rods.

Figure 4 shows measured transmittance and reflectance spectra for the sample shown in Figures 3b-d. The transmittance spectrum (Fig. 4a, right hand side, blue curve) shows high transmittance on the long-wavelength side with suppression down to $30 \%$ at a wavelength of $2.16 \mu \mathrm{m}$. The transmittance recovers on the short-wavelength side to almost $65 \%$, before higher bands and diffraction lead to a further reduction in transmittance. The reflectance spectrum (red curve) shows corresponding features. Band-structure calculations, ${ }^{[33]}$ with parameters taken from the electron microscopy images and especially taking into account the measured ellipsoidal rod cross section reveal a PBG of $3.5 \%$, marked by the hashed bar. At first sight, one might expect larger effects in the optical spectra. To allow for a direct comparison with the theoretical ideal, we have calculated corresponding spectra. We use a scattering-matrix approach ${ }^{[34]}$ with similar parameters used for the band-structure calculations but taking into account the finite size of the sample. The overall agreement between the calculations (dotted curves) and experimental results (solid curves) is very good. To mimic the experimental measurement apparatus (see Experimental), in particular the finite-opening angle due to the Cassegrain optics, we averaged the calculated transmittance spectra over an angle of incidence between 15 and $30^{\circ}$ with respect to the surface normal. Remaining discrepancies can be explained by structural imperfections of the sample. However, the effect of angle averaging is rather small. To show this, we also calculated the transmittance spectrum for normal incidence without any angle averaging (dashed grey curve). Recall that we have used similar structural parameters in both the scattering-matrix calculations (for the finite structure) and the band-structure calculations (for a fictitious infinite structure) and that 

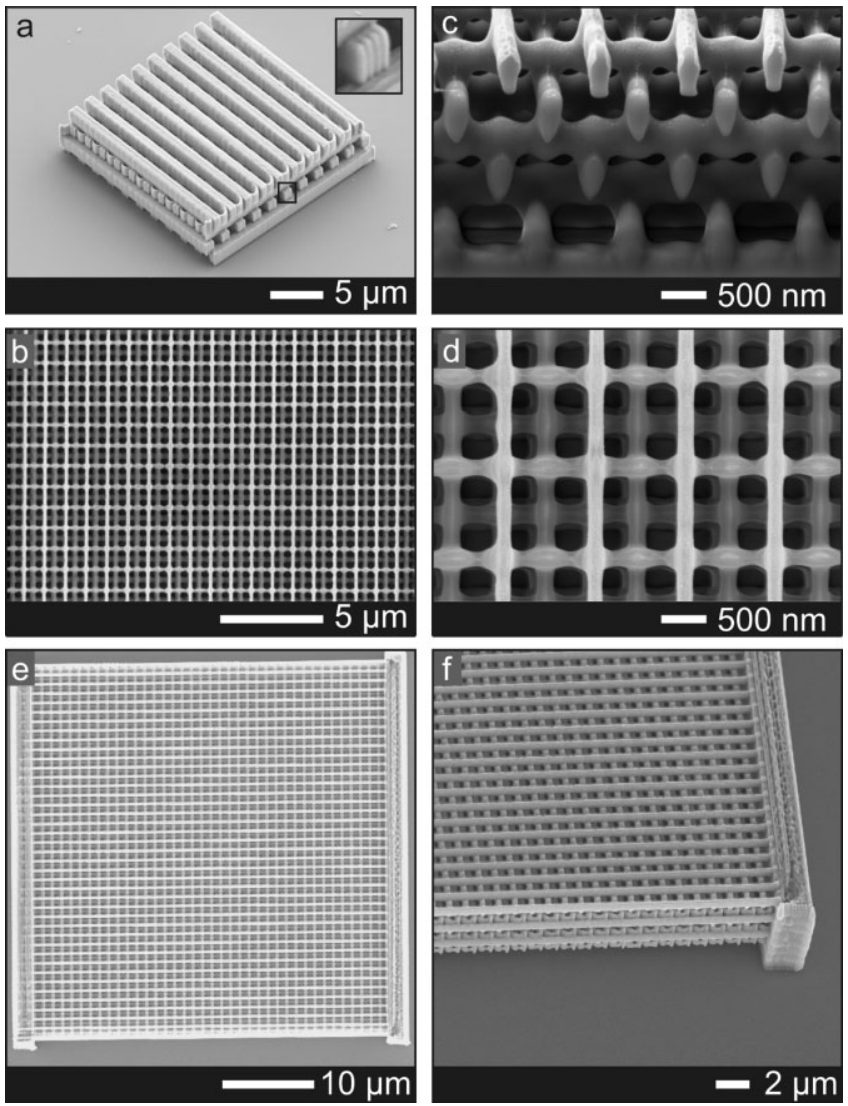

Figure 3. Scanning electron microscopy images of $\mathrm{As}_{2} \mathrm{~S}_{3}$ woodpiles. a) Woodpile with rod distance $a=2 \mu \mathrm{m}$ to illustrate the construction principle of the rods. Each rod is made from eight parallel subrods to yield a rod aspect ratio of almost 1.0 (see inset). b) Top view of a woodpile with rod distance $a=1 \mu \mathrm{m}$. Note the perfectly straight rods. c) Focused-ionbeam cross section of the woodpile in (b). d) Close up of (b). Note the smoothness of the rod surfaces. e) Top view of a woodpile similar to the one shown in (b) but with a $40 \mu \mathrm{m} \times 40 \mu \mathrm{m}$ footprint and twelve layers. f) Side view of the woodpile shown in (e). The walls merely serve as a support for the woodpile, which is intentionally raised off the substrate.

the latter reveal a complete PBG with $3.5 \%$ gap/midgap ratio. Thus, our experimental results are compatible with this PBG.

To demonstrate the potential of our approach, Figure $4 \mathrm{~b}$ presents a gap-width map, denoting the maximum achievable PBG for the parameters accessible by means of a modifiedrod design. The white cross marks the position for the sample presented. Further modification of the writing parameters might lead to a complete gap approaching a gap/midgap ratio of $9 \%$. As higher refractive index chalcogenide glasses, such as $\mathrm{As}_{2} \mathrm{Se}_{3}(n=2.78)$ or As-Se-Te composites ( $n$ up to 3.2), behave similarly under photoexposure, even larger PBGs could be achieved, if higher-order spherical aberrations are precompensated. In combination with the inherent ability of DLW to fabricate functional defects like waveguides and resonators, our approach might be a powerful alternative to established fabrication techniques.

In conclusion, we have demonstrated the direct fabrication of 3D, high-index-contrast nanostructures by DLW. Our approach uses high-index chalcogenide glasses and a newly
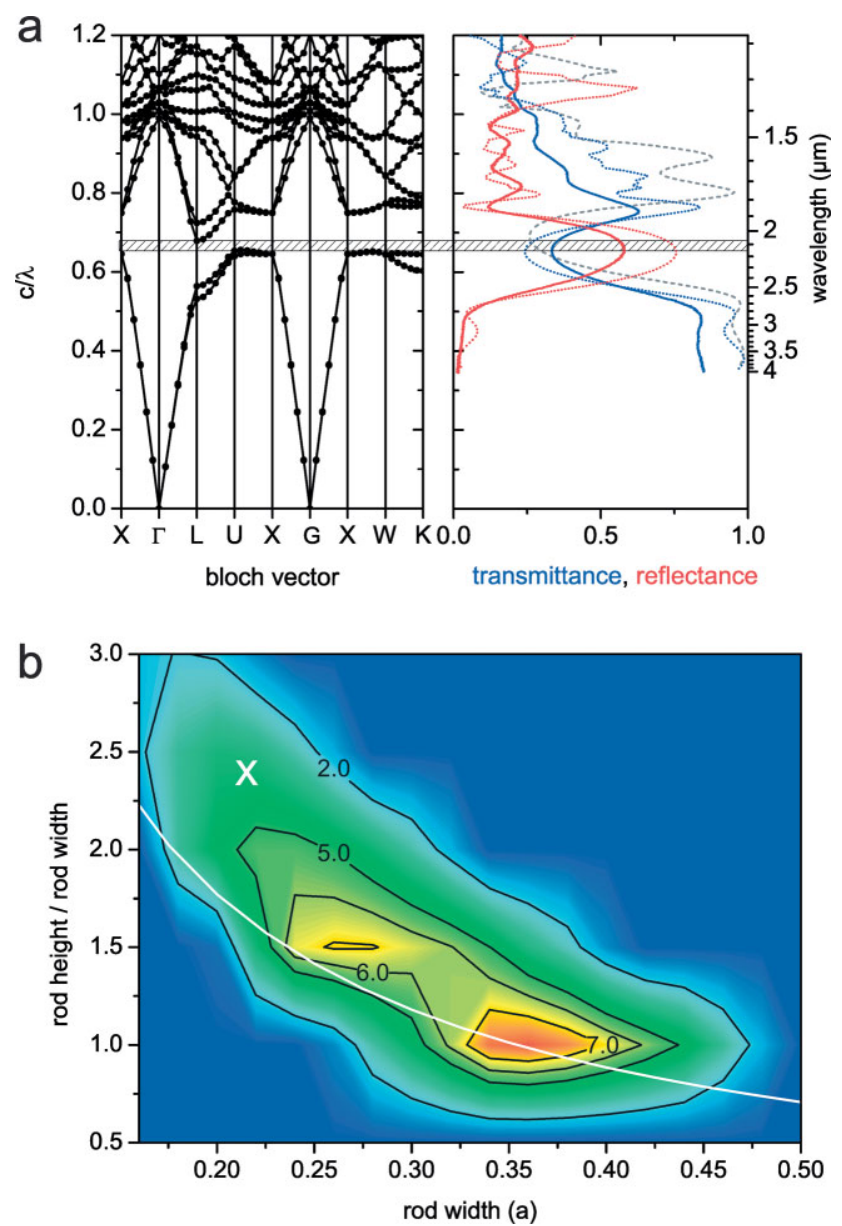

Figure 4. Optical characterization: a) comparison between band-structure calculation (left-hand side) and transmittance (right-hand side, blue solid curve) and reflectance spectra (right-hand side, red solid curve). The hashed area marks the complete gap in good correspondence with the optical spectra. The dotted curves are scattering matrix calculations, averaged over the experimental angles. The grey curve represents a calculation for perpendicular incidence without any angle averaging. b) Gapwidth map in percent of the mid-gap frequency for the independently adjustable parameters. The white cross marks the position corresponding to the sample measured in (a). Refining the parameters can yield a gap width of up to $8.8 \%$ for a rod aspect ratio of almost 1.0. Structures below the solid white line are not mechanically stable.

developed, highly selective etchant. We have given proof of concept by fabricating 3D woodpile photonic crystals compatible with a complete photonic bandgap of $3.5 \%$. This achievement has been made possible because we have compensated for a part of the distortions that are specific for writing into non-index-matched materials, i.e., for the defocus, the voxel elongation, and the use of a novel highly selective etchant. Useful photonic crystals can be fabricated along these lines in a total time of less than two hours. With future work aiming at further improvements regarding the correction of higher-order spherical aberrations, chalcogenide glasses promise to be a powerful alternative to common crystalline semiconductors in photonic-crystal fabrication, especially well suited for the near-infrared and possibly even for the visible spectral region. 


\section{Experimental}

For the fabrication of thin chalcogenide films we thermally evaporated glassy $\mathrm{As}_{2} \mathrm{~S}_{3}$ (Amorphous Materials Inc.) in a high-vacuum evaporation chamber at a temperature of $T=385^{\circ} \mathrm{C}$, under an evaporation pressure of $5 \times 10^{-6} \mathrm{mbar}(1 \mathrm{bar}=100000 \mathrm{~Pa})$, and with a rate, measured with a quartz microbalance, of approximately $6 \mathrm{~nm} \mathrm{~s}^{-1}$. $\mathrm{X}$-ray diffraction and Raman spectroscopy confirmed the presence of the molecular glass (not shown).

For DLW a regeneratively amplified Ti:sapphire laser system (SpectraPhysics Hurricane) was used with a pulse duration of 120 fs. The repetition rate could be computer controlled from $1 \mathrm{kHz}$ to single shots. The wavelength was tuned to $800 \mathrm{~nm}$, where one-photon absorption of the chalcogenide glass was negligible. The output beam was attenuated by a half-wave plate/polarizer combination; after beam expansion, typically $9.5 \mathrm{~nJ}$ of single pulse energy is coupled into an inverted microscope (Leica). The femtosecond pulses were focused into the chalcogenide film by a $100 \times$ oil-immersion objective with a high numerical aperture (NA=1.4, Leica). The sample was placed on a capacitively controlled, three-axis piezo scanning stage, which was operated in a closed loop providing a resolution of better than $5 \mathrm{~nm}$ at the full scanning range of $200 \mu \mathrm{m} \times 200 \mu \mathrm{m} \times 20 \mu \mathrm{m}$ (Physik Instrumente). The scanning operation of the piezo was computer controlled and its movements were synchronized with the output of the laser system.

All chemicals were used as received. Diisopentylamine (Alfa Aesar), with concentrations between 0.05 and 5.3 mol- $\%$ was used. This secondary amine molecule contained two sterically bulky organic groups attached to the nitrogen atom, making dissolution of the photopolymerized areas-crosslinked $\mathrm{As}_{2} \mathrm{~S}_{3}$ glassy network-more difficult, while maintaining the rate of dissolution of the smaller $\mathrm{As}_{4} \mathrm{~S}_{6}$ molecules in the unexposed regions. The amine was used with increasing dilution with polar organic solvents such as dimethylsulfoxide (Aldrich) so that the rate between the removal of the reaction products and the etching of the highly porous 3D structure could be effectively controlled. The overall effect of these two factors increases the selectivity of the etchant, allowing for the production of 3D structures with high aspect ratios and fine feature resolution.

Transmittance and reflectance spectra were measured using a nearinfrared Fourier-transform interferometer (Bruker Equinox 55, NIR halogen source) connected to an infrared microscope (Bruker Hyperion 1000, 36x Cassegrain lens, NA=0.5, liquid $\mathrm{N}_{2}$-cooled InSb detector). Due to the Cassegrain optics, the incident light spans an angle between 15 and $30^{\circ}$ with respect to the surface normal for "normal incidence", spanning part of the LUX (see Fig. 4a) directions. The optical axis corresponds to the $\Gamma \mathrm{X}$ direction for face-centered- cubic symmetry. The PC transmittance was normalized to the transmittance of the bare glass substrate. The reflectance was normalized to a silver mirror.

For the numerical calculations we used the freely available MIT photonic bands package [31] (band structure) and our own scattering matrix code (spectra). The required structural parameters for the band-structure calculations were obtained as follows: the rod aspect ratio was measured for all rods visible in Figure 2c, and an average value of 2.4 was then used for the calculations. The same procedure was applied to determine the rod diameter $(220 \mathrm{~nm})$. We used a rod distance of $1 \mu \mathrm{m}$ for calculation of the spectra, consistent with the rod distance found in Figures $3 \mathrm{c}-\mathrm{f}$. A refractive index of 2.5 and a perfect ellipsoidal shape of the rods were used in the calculations.

Received: September 16, 2005 Final version: November 9, 2005 Published online: January 10, 2006

[1] E. Yablonovitch, Phys. Rev. Lett. 1987, 58, 2059.

[2] S. John, Phys. Rev. Lett. 1987, 58, 2486.
[3] S. Y. Lin, J. G. Fleming, D. L. Hetherington, B. K. Smith, R. Biswas, K. M. Ho, M. M. Sigalas, W. Zzubrzycji, S. R. Kurtz, J. Bur, Nature 1998, 394, 251.

[4] S. Noda, K. Tomoda, N. Yamamoto, A. Chutinan, Science 2000, 289, 604.

[5] A. Feigel, Z. Kotler, B. Sfez, A. Arsh, M. Klebanov, V. Lyubin, Appl. Phys. Lett. 2000, 77, 3221.

[6] A. Feigel, M. Veinger, B. Sfez, A. Arsh, M. Klebanov, V. Lyubin, Appl. Phys. Lett. 2003, 83, 4480.

[7] R. Mayoral, J. Requene, J. S. Moya, C. Lopez, A. Cintas, H. Miguez, F. Meseguer, L. Vazquez, M. Holgado, A. Blanco, Adv. Mater. 1997, 9, 257.

[8] S. Wong, V. Kitaev, G. A. Ozin, J. Am. Chem. Soc. 2003, 125, 15589.

[9] F. Campbell, D. N. Sharp, M. T. Harrison, R. G. Denning, A. J. Turberfield, Nature 2000, 404, 53.

[10] Yu. V. Miklyaev, D. C. Meisel, A. Blanco, G. von Freymann, K. Busch, W. Koch, C. Enkrich, M. Deubel, M. Wegener, Appl. Phys Lett. 2003, 82, 1284 .

[11] S. Kawata, H.-B. Sun, T. Tanaka, K. Takada, Nature 2001, $412,697$.

[12] M. Deubel, G. von Freymann, M. Wegener, S. Pereira, K. Busch, C. M. Soukoulis, Nat. Mater. 2004, 3, 444

[13] J. Wijnhoven, W. L. Vos, Science 1998, 281, 802.

[14] A. Blanco, E. Chomski, S. Grabtchak, M. Ibisate, S. John, S. W. Leonard, C. Lopez, F. Meseguer, H. Miguez, J. P. Mondia, G. A. Ozin, O. Toader, H. M. van Driel, Nature 2000, 405, 437

[15] Y. A. Vlasov, X. Z. Bo, J. C. Sturm, D. J. Norris, Nature 2001, 414, 289.

[16] H. Míguez, Adv. Mater. 2001, 15, 567.

[17] N. Tétreault, G. von Freymann, M. Deubel, M. Hermatschweiler, F. Pérez-Willard, S. John, M. Wegener, G. A. Ozin, Adv. Mater, October 11, 2005, DOI: 10.1002/adma.200501674

[18] K.-M. Ho, C. T. Chan, C. M. Soukoulis, R. Biswas, M. Sigalas, Solid State Comm. 1994, 89, 413.

[19] Although 3D photonic crystals with a complete gap can also be fabricated based on macroporous silicon, the introduction of arbitrarily shaped functional defects is conceptually difficult. See S. Matthias, F. Müller, C. Jamois, R. B. Wehrspohn, U. Gösele, Adv. Mater. 2004, 16, 2166.

[20] J. P. De Neufville, S. C. Moss, S. R. Ovshinsky, J. Non-Cryst. Solids 1973/74, 13, 191.

[21] A. V. Kolobov, Photo-induced Metastability in Amorphous Semiconductors, Wiley-VCH Weinheim, Germany 2003

[22] P. V. Braun, R. W. Zehner, C. A. White, M. K. Weldon, C. Kloc, S. S. Patel, P. Wiltzius, Adv. Mater. 2001, 13, 721.

[23] V. N. Astratov, A. M. Adawi, M. S. Skolnick, V. K. Tikhomirov, V. Lyubin, D. G. Lidzey, M. Ariu, A. L. Reynolds, Appl. Phys. Lett. 2001, 78, 4094.

[24] B. H. Juarez, M. Ibisate, J. M. Palacios, C. Lopez, Adv. Mater. 2003, 15,319 .

[25] M. Hammam, J. J. Santiago, Solid State Comm. 1986, 59, 725.

[26] K. Tanaka, Y. Ohtsuka, Thin Solid Films 1979, 57, 59.

[27] K. White, B. Kumar, A. K. Rai, Thin Solid Films 1988, 161, 139.

[28] M. Janai, P. S. Rudman, A. Mandelbaum, J. Non-Cryst. Solids 1978, $27,67$.

[29] S. A. Solin, G. N. Papatheodorou, Phys. Rev. B 1977, 15, 2084.

[30] A. J. Bansiter, I. Gorrell, Adv. Mater. 1998, 10, 1415.

[31] P. Török, P. Varga, Y. Lacyik, G. R. Booker, J. Opt. Soc. Am. A 1995, 12,325 .

[32] C. J. R. Sheppard, M. Gu, Appl. Opt. 1991, 30, 3563.

[33] We use the freely available MIT photonic bands package. For details see: S. G. Johnson, J. D. Joannopoulos, Optics Express 2001, 8,173 .

[34] D. M. Whittaker, I. S. Culshaw, Phys. Rev. B 1999, 60, 2610. 